\title{
Properties of short channel ballistic carbon nanotube transistors with ohmic contacts
}

\author{
François Léonard* \\ Sandia National Laboratories, Livermore, CA 94551 \\ Derek A. Stewart ${ }^{\dagger}$ \\ Cornell Nanoscale Science and Technology Facility, Ithaca, NY 14853
}

(Dated: November 2, 2006)

\begin{abstract}
We present self-consistent, non-equilibrium Green's function calculations of the characteristics of short channel carbon nanotube transistors, focusing on the regime of ballistic transport with ohmic contacts. We first establish that the band lineup at the contacts is renormalized by charge transfer, leading to Schottky contacts for small diameter nanotubes and ohmic contacts for large diameter nanotubes, in agreement with recent experiments. For short channel ohmic contact devices, source-drain tunneling and drain-induced barrier lowering significantly impact the current-voltage characteristics. Furthermore, the ON state conductance shows a temperature dependence, even in the absence of phonon scattering or Schottky barriers. This last result also agrees with recently reported experimental measurements.
\end{abstract}

\section{INTRODUCTION}

Since their original fabrication ${ }^{1,2}$, carbon nanotube (NT) transistors have seen much improvement in their performance $^{3}$, and in the understanding of the science that governs their operation. For example, it has recently been demonstrated that the metal used to contact the NT has a strong influence on the device behavior, with reports of ohmic contacts for $\mathrm{Pd}^{4,5}$ and $\mathrm{Au}^{6}$, and Schottky contacts for $\mathrm{Ti}^{7}$. Furthermore, it has been demonstrated that scaling of Schottky barrier NT transistors is very different from traditional transistors ${ }^{8}$. These studies highlight the danger of using concepts from conventional silicon devices and applying them to NT devices. In such circumstances, modeling can play a key role in predicting device behavior, and in supporting experimental conclusions.

While some theoretical and modeling work beyond traditional transistor models has been done to study NT transistors, these calculations have used various approximations, either as simplified treatment of the NT electronic properties ${ }^{8}$, or by simplifying the calculation of the current at finite bias voltage ${ }^{8,9}$. These approximations prevent a more detailed and quantitative study of the properties of NT devices, particularly under nonequilibrium conditions. Here, we present a full selfconsistent calculation that treats the NT electronic structure and the non-equilibrium quantum electron transport from an atomistic perspective. This allows the calculation of the charge, electrostatic potential and current in the device from a quantum transport approach, and includes quantum effects such as energy quantization and tunneling. It also naturally captures distortions of the NT bandstructure due to the spatially-varying electrostatic potential and is amenable to extensions to include scattering with defects, surfaces, phonons, etc.

The calculations presented here provide an approach for evaluating the performance of ultimate NT transistors, those with ballistic transport, ohmic contacts and nanometer channels (a recent paper presented a similar approach ${ }^{10}$, but focused on Schottky barrier NT transistors). We show that side contacts between NTs and metal are governed by charge transfer and band re-alignment, leading to a NT diameter above which contacts are ohmic, in agreement with recent experiments ${ }^{11,12}$. For the ohmic contact devices, short channel effects can dominate the device behavior for relatively large channel to gate length ratios. Furthermore, while the temperature dependence of the ON state conductance in NT devices can be dominated by Schottky contacts or phonon scattering, we show that, even in the absence of these effects, the conductance can have a strong temperature dependence. In fact, we find that the ON state conductance decreases with increasing temperature, in contrast to Schottky barrier NT transistors and in agreement with recent experimental measurements on Pd-contacted NT transistors ${ }^{4}$.

\section{MODELING APPROACH}

As shown in figures 1 and 2, the system consists of an infinitely long single-wall zigzag NT embedded in metal at its two ends and coated by a dielectric in the channel region. The gate insulator dielectric constant $\varepsilon$ is equal to 3.9 , as for $\mathrm{SiO}_{2}$, and in this work the dielectric is wrapped by a cylindrical gate of radius $3 \mathrm{~nm}$ in the channel region (unless otherwise stated). The nanotube sits in the middle of a smooth cylindrical hole in the metal and dielectric, and we take a spacing of $s=0.3$ $\mathrm{nm}$ between the NT and the metal or dielectric walls. The length of each contact $\lambda$ is equal to $4.17 \mathrm{~nm}$, sufficiently long to converge the electrostatic potential in the contacts.

The electronic properties of the NT are described using a tight-binding framework with one $\pi$ orbital per carbon atom, and a coupling $\gamma=2.5 \mathrm{eV}^{13}$ between nearestneighbor atoms. In our calculations, we assume that the electronic structure of the NT is not perturbed by atomic interactions with the metal or the dielectric. 


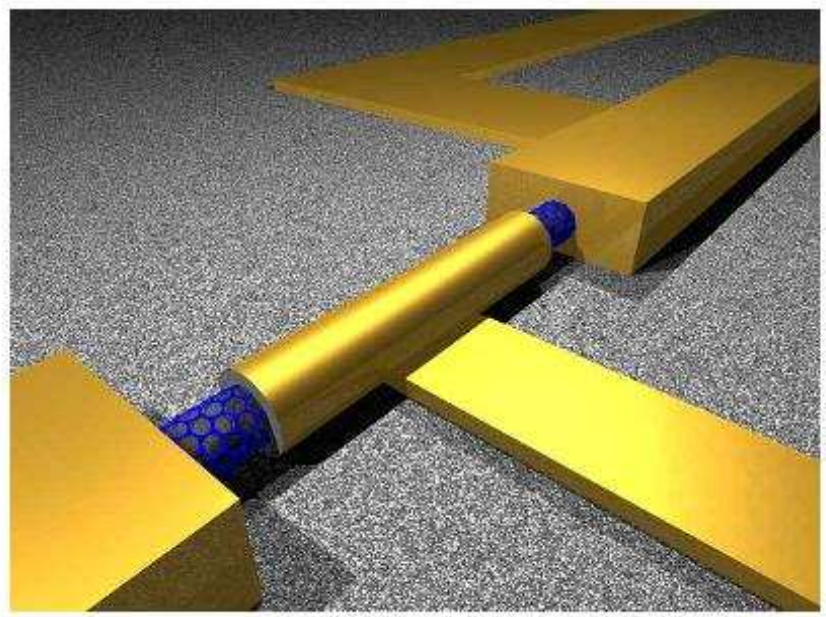

FIG. 1: Sketch of the NT transistor. The NT is embedded in metals at its two ends, and in a dielectric in the channel region. The dielectric is wrapped by a cylindrical gate of $3 \mathrm{~nm}$ radius. The separation between the contacts and the central dielectric region is to illustrate the structure in the channel; in the calculations, the contacts touch the dielectric.

To calculate the electronic current flowing through the NT, we apply the non-equilibrium Green's function formalism ${ }^{14}$ to the NT device, dividing the NT in principle layers, with each layer corresponding to a ring of the zigzag NT. The device is divided into a scattering region (layers 1 to $N$ ) connecting the lead regions, which consist of semi-infinite nanotubes embedded in the source and drain contact metals. This approach is illustrated in Fis ?

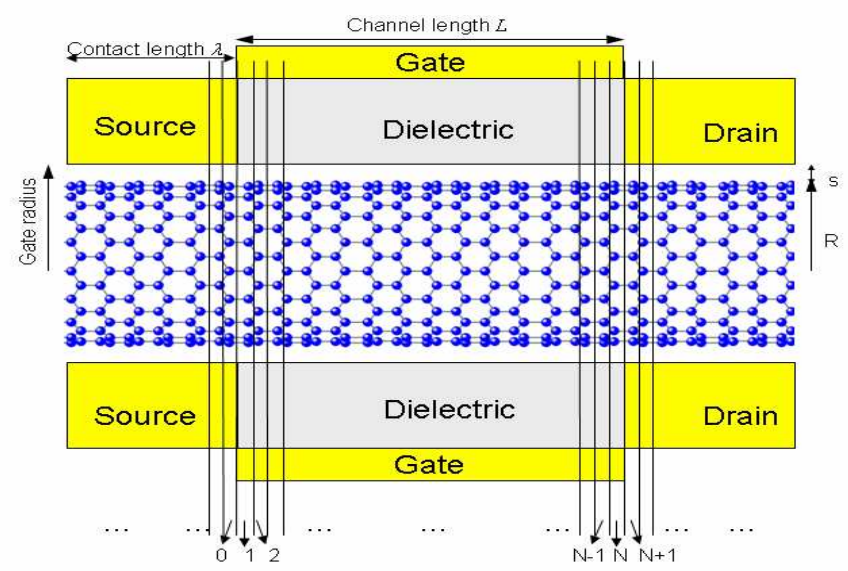

FIG. 2: Sketch of a cross-section of the nanotube transistor, showing the important device dimensions and the principle layers used for the quantum transport calculations.

For this geometry, the current can be obtained from

$$
I=\frac{4 e \gamma}{\pi \hbar} \int d E \operatorname{Re} G_{N, N+1}^{<}
$$

where $G^{<}$is the Green's function, indexed according to the principle layers in the device, with $N$ total layers in the scattering region. $G^{<}$is calculated by solving the matrix equations

$$
G^{<}=G^{R} \Sigma^{<} G^{R \dagger}
$$

and

$$
G^{R}=\left[(E-e U) I-H_{0}-\Sigma_{L}^{R}-\Sigma_{R}^{R}\right]^{-1},
$$

where $H_{0}$ is the tight-binding Hamiltonian for the isolated NT and $U$ is the electrostatic potential on each layer of the system. In our tight-binding representation, the Hamiltonian matrix elements are $H_{0}^{2 p, 2 p-1}=H_{0}^{2 p-1,2 p}=$ $2 \gamma \cos \left(\frac{\pi J}{M}\right), H_{0}^{2 p, 2 p+1}=H_{0}^{2 p+1,2 p}=\gamma$ where $M$ is the number of atoms around a NT ring and $J=1, \ldots, M$ labels each of the NT bands. Because of the cylindrical symmetry, we take the electrostatic potential on every atom of a ring to be the same, allowing substantial reduction in the size of the matrices (from $M N \times M N$ to $N \times N)$. The functions $\Sigma_{L, R}^{R}$ and $\Sigma^{<}$represent the interaction of the scattering region with the semi-infinite NT left $(L)$ and right $(R)$ leads. These functions are given by

$$
\Sigma_{L, R}^{R}=\tau_{L, R}^{\dagger} g_{L, R}^{R} \tau_{L, R}
$$

and

$$
\Sigma^{<}=-2 i \operatorname{Im}\left(f_{L} \Sigma_{L}^{R}+f_{R} \Sigma_{R}^{R}\right)
$$

where $\tau_{L, R}$ is the matrix that couples the scattering region to the left and right leads, respectively, $g_{L, R}^{R}$ is the Green's function for the semi-infinite left and right leads and $f_{L, R}$ are the left and right fermi functions. For the zigzag NT in Fig. 2, and within a nearest-neighbor tightbinding representation, only the first and last rings couple with the leads. Therefore, the coupling matrices $\tau_{L, R}$ each have only one non-zero element, equal to $\gamma$, and we have the non-zero components

$$
\begin{aligned}
{\left[\Sigma_{L}^{R}\right]_{11} } & =\gamma^{2}\left[g_{L}^{R}\right]_{00} \\
{\left[\Sigma_{R}^{R}\right]_{N N} } & =\gamma^{2}\left[g_{R}^{R}\right]_{N+1 N+1}
\end{aligned}
$$

where the indices of $g_{L}^{R}\left(g_{R}^{R}\right)$ run from $-\infty$ to 0 $(N+1$ to $\infty)$. The surface Green's functions $g_{L, R}^{R}$ are calculated using an iterative layer doubling technique ${ }^{15}$, while $G^{R}$ is calculated by using an efficient approach that iteratively builds up the Green's function at each layer across the device region ${ }^{16}$.

The electrostatic potential is calculated by solving Poisson's equation with a spatially-dependent dielectric constant $\kappa$

$$
\nabla \cdot(\kappa \nabla U)=-\frac{1}{4 \pi \varepsilon_{0}} \sigma
$$

in three-dimensional cylindrical coordinates on a grid with the source charge on the NT $\sigma$, and with boundary 
conditions at the source, drain, and gate surfaces, and at the boundaries of the simulation cell. We model the interface between the oxide and vacuum with the function
$2 \kappa(r)=(\varepsilon+1)+(\varepsilon-1) \tanh [(r-R-s) / \xi]$ where $\xi$ is a length scale on the order of a lattice constant.

In cylindrical coordinates, the above equation is

$$
\frac{\partial^{2} U(r, \phi, z)}{\partial r^{2}}+\frac{1}{r} \frac{\partial U(r, \phi, z)}{\partial r}+\frac{\partial^{2} U(r, \phi, z)}{\partial z^{2}}+\frac{1}{\kappa(r)} \frac{\partial \kappa(r)}{\partial r} \frac{\partial U(r, \phi, z)}{\partial r}=-\frac{1}{4 \pi \varepsilon_{0} \kappa(r)} \sigma(r, \phi, z)
$$

with the boundary conditions

$$
\begin{aligned}
U(r & >R+s, \phi, z \leq-L / 2)=0 \\
U(r & >R+s, \phi, z \geq L / 2)=V_{d s} \\
U(r & \left.=R_{g}, \phi,-L / 2<z<L / 2\right)=V_{g s} \\
\left.\frac{d U(r, \phi, z)}{d r}\right|_{r=0} & =0 \\
\left.\frac{d U(r, \phi, z)}{d z}\right|_{z=0} & =0 \\
\left.\frac{d U(r, \phi, z)}{d z}\right|_{z=L+2 \lambda} & =0
\end{aligned}
$$

where $L$ is the channel length, $V_{d s}$ is the drain-source voltage and $V_{g s}$ is the gate-source voltage. As will be discussed below, we use a uniform distribution of the charge in the azimuthal direction, thus reducing the three-dimensional partial differential equation to a twodimensional partial differential equation in $r$ and $z$. To solve this two-dimensional equation numerically, we use a finite-difference scheme

$$
\frac{U_{i+1, j}+U_{i-1, j}-2 U_{i, j}}{\Delta_{r}^{2}}+\frac{1}{r_{i}} \frac{U_{i+1, j}-U_{i-1, j}}{2 \Delta_{r}}+\frac{U_{i, j+1}+U_{i, j-1}-2 U_{i, j}}{\Delta_{z}^{2}}+\frac{1}{\xi} \frac{\varepsilon-1}{\varepsilon} \frac{U_{i_{c}+1, j}-U_{i_{c}, j}}{\Delta_{r}}=-\frac{1}{4 \pi \varepsilon_{0} \kappa_{i, j}} \sigma_{i, j}
$$

where the subscripts $i, j$ denote a function evaluated at position $\left(r_{i}, z_{j}\right)$ with $r_{i}=(i-1) \Delta_{r}$ and $z_{j}=(j-1) \Delta_{z}$, and $i_{c}=(R+s) / \Delta_{r}$ is the radial grid point where the contacts and dielectric begin. The dielectric/vaccum boundary term was evaluated using a forward difference scheme, assuming that $\xi \ll \Delta_{r}$; in practice we use a value $\xi=0.0085 \mathrm{~nm}$ and we verified that the ratio of the electric fields equals the ratio of the dielectric constants at the interface. The finite-difference boundary conditions are

$$
\begin{aligned}
U_{i \geq i_{c}, j \leq j_{S}} & =0 \\
U_{i \geq i_{c}, j \geq j_{D}} & =V_{d s} \\
U_{i_{G}, j_{S}<j<j_{D}} & =V_{g s} \\
U_{1, j} & =U_{2, j} \\
U_{i, 1} & =U_{i, 2} \\
U_{i, j_{D}+j_{S-1}} & =U_{i, j_{D}+j_{S}}
\end{aligned}
$$

where $j_{S}=\lambda / \Delta_{z}$ is the axial grid point where the source contact ends, $j_{D}=(L+\lambda) / \Delta_{z}$ is the axial grid point where the drain contact begins, and $i_{G}$ is the radial grid point at the gate radius. In our calculations we use grid spacings of $\Delta_{z}=0.07 \mathrm{~nm}$ in the axial direction and $\Delta_{r}=0.03 \mathrm{~nm}$ in the radial direction. Numerical solution of Eq. (10) is obtained using a successive over-relaxation procedure $^{17}$. Once the electrostatic potential is obtained, the value for $U$ on each layer of the system (i.e. that enters the Hamiltonian) is taken as the value of the electrostatic potential at the atomic position of each ring along the NT.

To obtain the charge density, we note that the tightbinding technique only provides the total charge on each layer; in our formalism the total charge on layer $l$ is given by $\frac{e}{2 \pi} \int d E \operatorname{Im} G_{l l}^{<}$, which needs to be spatially distributed. We assume a uniform distribution of the charge in the azimuthal direction, and spatially distribute the total charge in the radial and axial directions with a Gaussian smearing function. Thus the three-dimensional charge density is given by

$$
\sigma(r, \phi, z)=\sum_{l} g\left(z-z_{l}, r-R\right) \frac{e}{2 \pi} \int d E \operatorname{Im} G_{l l}^{<}
$$

where $g\left(z-z_{l}, r-R\right)=\left(4 \pi^{2} R \sigma_{z} \sigma_{r}\right)^{-1}$ $\exp \left[-\left(z-z_{l}\right)^{2} / 2 \sigma_{z}^{2}\right] \exp \left[-(r-R)^{2} / 2 \sigma_{r}^{2}\right]$ with $R$ the tube radius, $z_{l}$ the position of ring $l$, and $\sigma_{z}$ and $\sigma_{r}$ the smearing lengths in the axial and radial directions respectively (this expression for $g$ is valid when $R \gg \sigma_{r}$, and we use values of $\sigma_{z}=0.14 \mathrm{~nm}$ and $\sigma_{r}=0.06 \mathrm{~nm}$ ).

Our overall procedure is to start from some initial guess for the potential and solve for the Green's functions from 
the system of Eqs $(2-6)$, calculate the charge distril tion in the device from Eq. 12, and then re-calculate $t$ potential profile across the device based on solving $\mathrm{Pc}$ son's equation. This process is iterated until both $t$ potential and charge distribution converge; in pract this is done using a simple mixing of the charge at ec iteration step.

As discussed in a previous work ${ }^{9}$, short channel I transistors can possess two different operating regimes conventional transistor regime, and a Coulomb blocke regime at large gate voltages due to channel inversi While simplified models exist to describe the Coulor blockade regime ${ }^{9}$, a proper treatment involves includi the electron-electron interaction self-consistently in $t$ Green's function approach. In this paper, we focus the transistor regime and leave the Coulomb blocke regime for future work.

\section{PROPERTIES OF CONTACTS}

Before discussing the full current-voltage character tics of the NT transistor, we begin by discussing the prc erties of the contacts for NTs embedded in metals. F 3 shows a cross-section of the contact, and the asso ated bare band lineup. For such a contact, the differer between the metal Fermi level $E_{F}$ and the valence ba edge $E_{v}$ before charge transfer is simply given by

$$
E_{F}-E_{v}=\Phi_{N T}-\Phi_{m}+\frac{1}{2} E_{g}
$$

where $\Phi_{m}$ and $\Phi_{N T}$ are the metal and NT workfunctions respectively, and $E_{g}$ is the NT band gap. A positive value for $E_{F}-E_{v}$ indicates a Schottky barrier, while a negative value indicates an ohmic contact (here we focus on the barrier for holes, the relevant quantity for high workfunction metals used in most experiments; the barrier for electrons is $E_{v}+E_{g}-E_{F}$ and would be relevant for low workfunction metals. Because the NT band structure is symmetric around the midgap, the results presented below apply to both cases). Given the relation $E_{g}=a \gamma / d$ between bandgap and NT diameter $d(a=0.142 \mathrm{~nm}$ is the $\mathrm{C}-\mathrm{C}$ bond length), the band lineup thus depends on the NT diameter. The behavior of Eq. 13 is shown in Fig. 4 for two different values of $\Phi_{m}-\Phi_{N T}$ as a function of the NT diameter. This simple picture for the band lineup is modified due to charge transfer between the metal and NT, as we now discuss.

Because the metal Fermi level is not in line with the charge neutrality level in the NT (midgap for an undoped NT), charge transfer between the metal and NT occurs, as illustrated in Fig. 3a. From an image potential argument, this transferred charge creates a charge dipole at the nanotube/metal interface, and an associated electrostatic potential. This electrostatic potential in turn shifts the bands, and changes the amount of transferred charge. Thus, the charge and potential must be determined self-consistently. This behavior can be described (a)

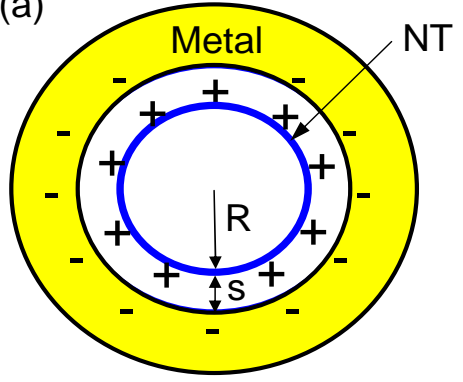

(b)

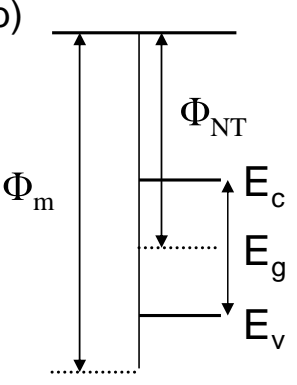

FIG. 3: Panel (a) shows a sketch of the cross-section of the nanotube/metal contact, while panel (b) is the bare band lineup.

using simple models for the charge and the potential (the results of the atomistic approach presented in the previous section will be discussed further below). The charge per atom on the NT can be expressed as

$$
\sigma=e N \int D(E) f\left(E-E_{F}\right) d E
$$

where

$$
D(E)=\frac{a \sqrt{3}}{\pi^{2} R \gamma} \frac{\left|E+e V_{N T}\right|}{\sqrt{\left(E+e V_{N T}\right)^{2}-\left(E_{g} / 2\right)^{2}}}
$$

is the density of states, $f\left(E-E_{F}\right)$ is the Fermi function and $N=4 /\left(3 \sqrt{3} a^{2}\right)$ is the atomic areal density. We assume a uniform distribution of the charge on the NT.

For the geometry of Fig. 3a, solution of Poisson's equation gives the potential on the NT as

$$
e V_{N T}=-\sigma \frac{e R}{\varepsilon_{0}} \ln \frac{R+s}{R} .
$$

For a given NT, equations 14 and 16 can be solved selfconsistently. The solid lines in Fig. 4 show results of such calculations for the room temperature alignment between the metal Fermi level and the NT band edge for $\Phi_{m}-\Phi_{N T}=1 \mathrm{eV}$ and $0.3 \mathrm{eV}$. Comparison with the barrier predicted from Eq. 13 (dotted lines in the figure) indicates that the charge transfer significantly changes the band line-up. While for the higher workfunction difference the contacts are ohmic down to very small diameter NTs (Fig. 4a), for the lower workfunction metal (Fig. $4 \mathrm{~b}$ ), the results indicate a transition between Schottky and ohmic behavior at a NT diameter around $1.8 \mathrm{~nm}$. This result agrees with recently published experimental data $^{11,12}$.

The results of this simplified analytical model can be compared with calculations from the atomistic, selfconsistent, Green's function approach introduced in the previous section. For this comparison, the electrostatic potential on the NT from the atomistic approach is taken to be $U_{i_{R}, 1}$ where $i_{R}=R / \Delta_{r}$ is the grid point at the NT 

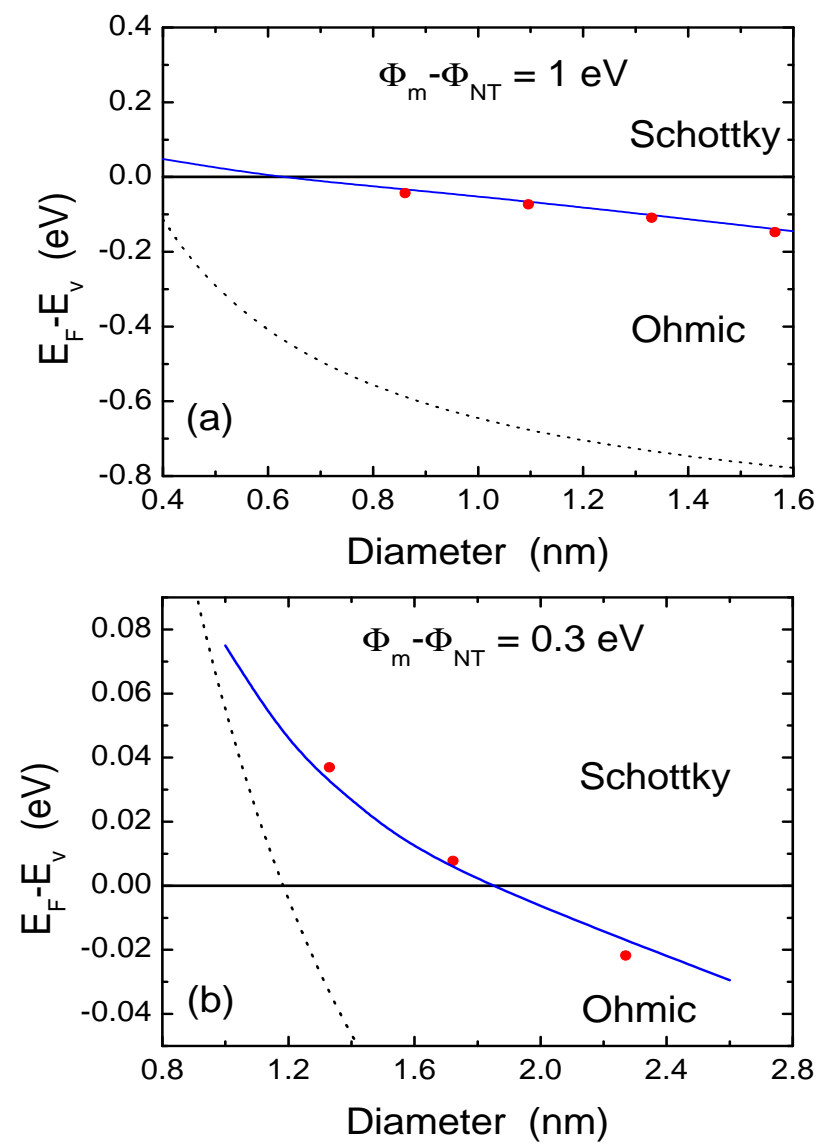

FIG. 4: Band lineup at nanotube/metal contacts. Dotted line is prediction from Eq. (13), solid line is computed from Eqs (14) and (16), and circles are calculated from atomistic approach.

radius and we use $V_{s d}=V_{g s}=0$ (since the contact length is long enough to screen the electric fields from the drain or gate electrodes, the value of $U_{i_{R}, 1}$ is independent of the choice of $V_{d s}$ and $V_{g s}$ ). The data points in Figure 4 show results of such calculations for several semiconducting zigzag NTs, demonstrating excellent agreement with the simplified approach presented above.

\section{TRANSISTOR CHARACTERISTICS}

Having established the conditions needed to make ohmic contacts to NTs, we now discuss the roomtemperature behavior of NT transistors with such contacts. For explicit calculations, we take the NT midgap as the energy reference level, take $\Phi_{m}-\Phi_{N T}=1 \mathrm{eV}$, and use a $(17,0)$ zigzag NT. This NT has a radius of $0.66 \mathrm{~nm}$ and in our tight-binding description, it has a bandgap of $0.55 \mathrm{eV}$.

The current versus gate-source voltage characteristics of the NT transistor calculated using the atomistic approach of section II are shown in Fig. 5 for two different values of the channel length, and for source-drain voltages of -0.1 and $-0.3 \mathrm{~V}$. The general behavior consists of an $\mathrm{ON}$ state at smaller values of $V_{g s}$ and an OFF state at larger values of $V_{g s}$. These two regimes originate from the presence or absence of a barrier blocking the hole current, as shown in the band diagrams of Fig. 5b. The $10 \mathrm{~nm}$ channel device shows rather poor characteristics, with an ON/OFF ratio of only 1000 and a subthreshold swing $S=\left(d \log I / d V_{g s}\right)^{-1}=160 \mathrm{mV} /$ decade. This behavior originates from poor control of the gate over the electrostatics in the channel, and from tunneling across the hole barrier in the OFF regime. Increasing the channel length to $20 \mathrm{~nm}$ as in Fig. 5b gives a much better $S=69 \mathrm{mV} /$ decade and improved ON/OFF ratio. A long-channel transistor with undoped channel is expected to be maximally OFF at $V_{g s}=0$; it is clear from Fig. 5 that even the $20 \mathrm{~nm}$ device is $\mathrm{ON}$ at that gate voltage. The inset in Fig. 5b shows the calculated band bending for $V_{g s}=0$ and $V_{d s}=0$, indicating that the channel is effectively doped $p$-type due to a long-ranged charge transfer from the contacts ${ }^{18}$. Thus the NT device is normally ON, despite the fact that the channel is undoped.
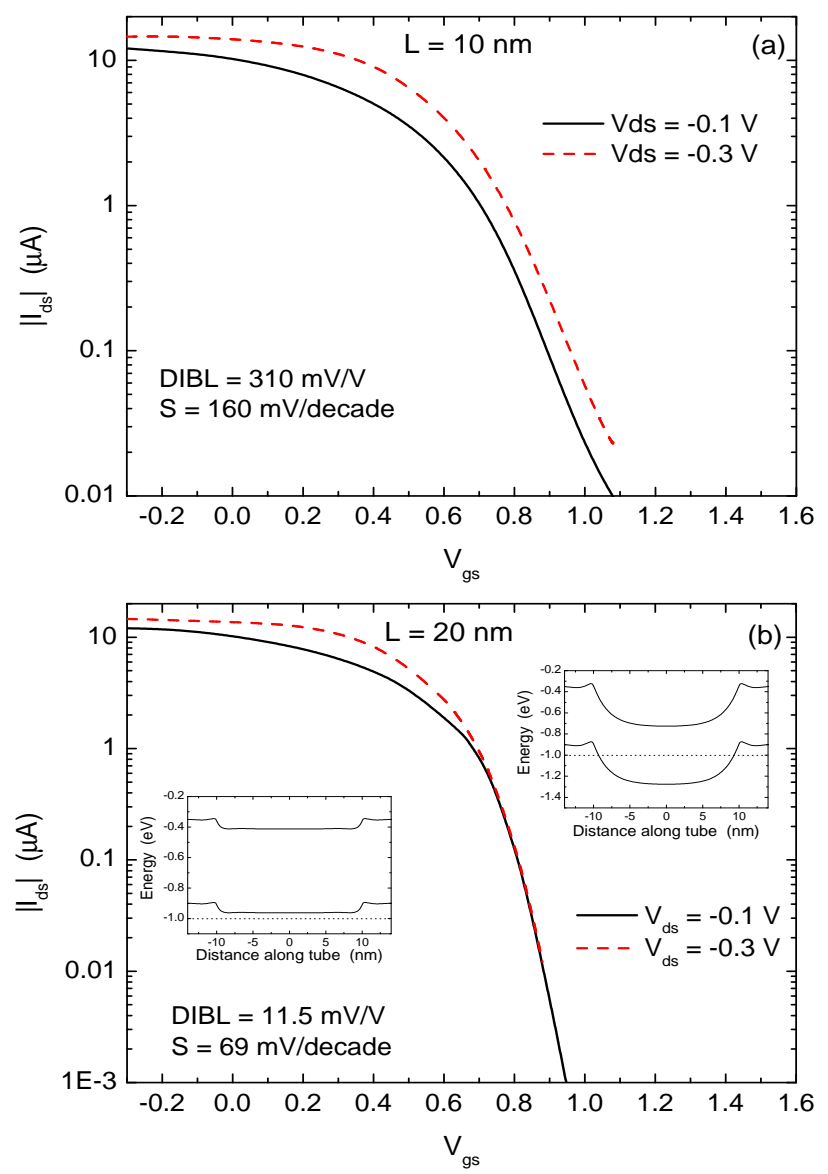

FIG. 5: Current as a function of gate-source voltage for channel lengths of (a) $10 \mathrm{~nm}$ and (b) $20 \mathrm{~nm}$. The insets show the band bending for $V_{g s}=0$ (left) and $V_{g s}=1 \mathrm{~V}$ (right).

The output characteristics of the NT transistor are 
shown in Fig. 6, indicating good saturation of the current for the $20 \mathrm{~nm}$ channel device, but no saturation for the $10 \mathrm{~nm}$ device. This behavior can be attributed to draininduced barrier lowering (DIBL) as indicated in Fig. 7. There, it is shown that for the $10 \mathrm{~nm}$ channel device, application of a source-drain voltage reduces the barrier between the source Fermi level and the middle of the channel. A signature of the DIBL effect is also seen in Fig. 5a, where there is significant shift between the transfer characteristics for source-drain voltages of -0.1 and -0.3 Volts. A quantitative calculation of the DIBL from $\Delta I / \Delta V_{d s}$ at $0.1 \mu A$ gives a very large value of $310 \mathrm{mV} / \mathrm{V}$. Fig. $5 \mathrm{~b}$ shows that the DIBL for the $20 \mathrm{~nm}$ channel length is reduced substantially to $11.5 \mathrm{mV} / \mathrm{V}$, consistent with the saturation of the output characteristics of Fig. 6.
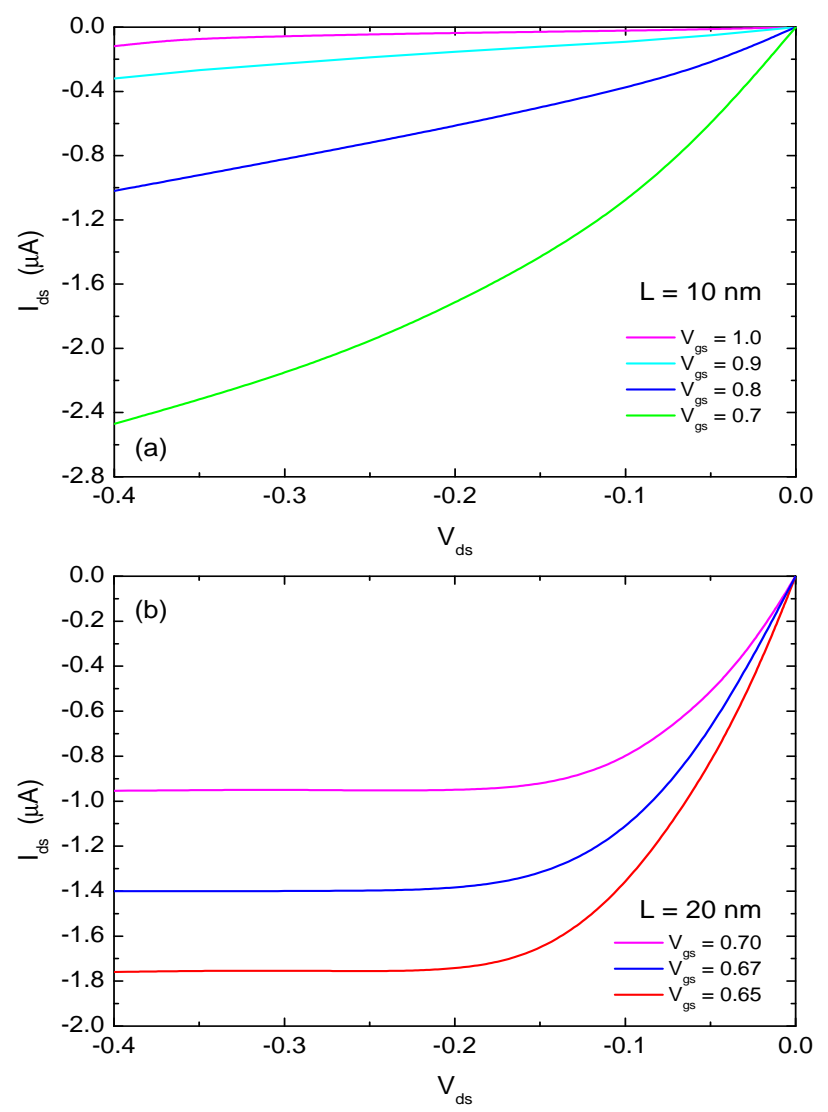

FIG. 6: Current as a function of drain-source voltage for (a) $10 \mathrm{~nm}$ channel length and (b) $20 \mathrm{~nm}$ channel length.

The reduction of the subthreshold swing and the DIBL with increasing channel length is captured in Fig. 8. It is clear that both quantities vary rapidly over the same length scale of a few nanometers. Typical device constraints require DIBL less than $100 \mathrm{mV} / \mathrm{V}$ and $S$ less than $80 \mathrm{mV} / \mathrm{dec}$; this restricts the nanotube transistor in this geometry and with this dielectric constant to a channel length greater than 15 nanometers, outside of the shaded areas in the figure. To extend this analysis, we repeated the calculations of the subthreshold swing for gate radii of 1.5, 6 and $9 \mathrm{~nm}$; the inset in Fig. 8a shows that a good scaling behavior can be obtained if the channel length is scaled by a quantity $\alpha \sim \sqrt{R_{g}+l}$, where $l=1 \mathrm{~nm}$. While we have not been able to derive an analytical expression to justify this scaling behavior, we note that electrostatic analyses for cylindrical gate transistors ${ }^{19,20}$ predict a scaling quantity proportional to the oxide thickness $\alpha \sim\left(R_{g}-R-s\right)$; we have tried this type of relation and find that it does collapse the data onto a single curve. We suspect that the unusual dielectric response of the $\mathrm{NT}^{21}$, strong charge transfer from the contacts and the actual device geometry render the conventional analyses inapplicable; more work is needed to address these issues.
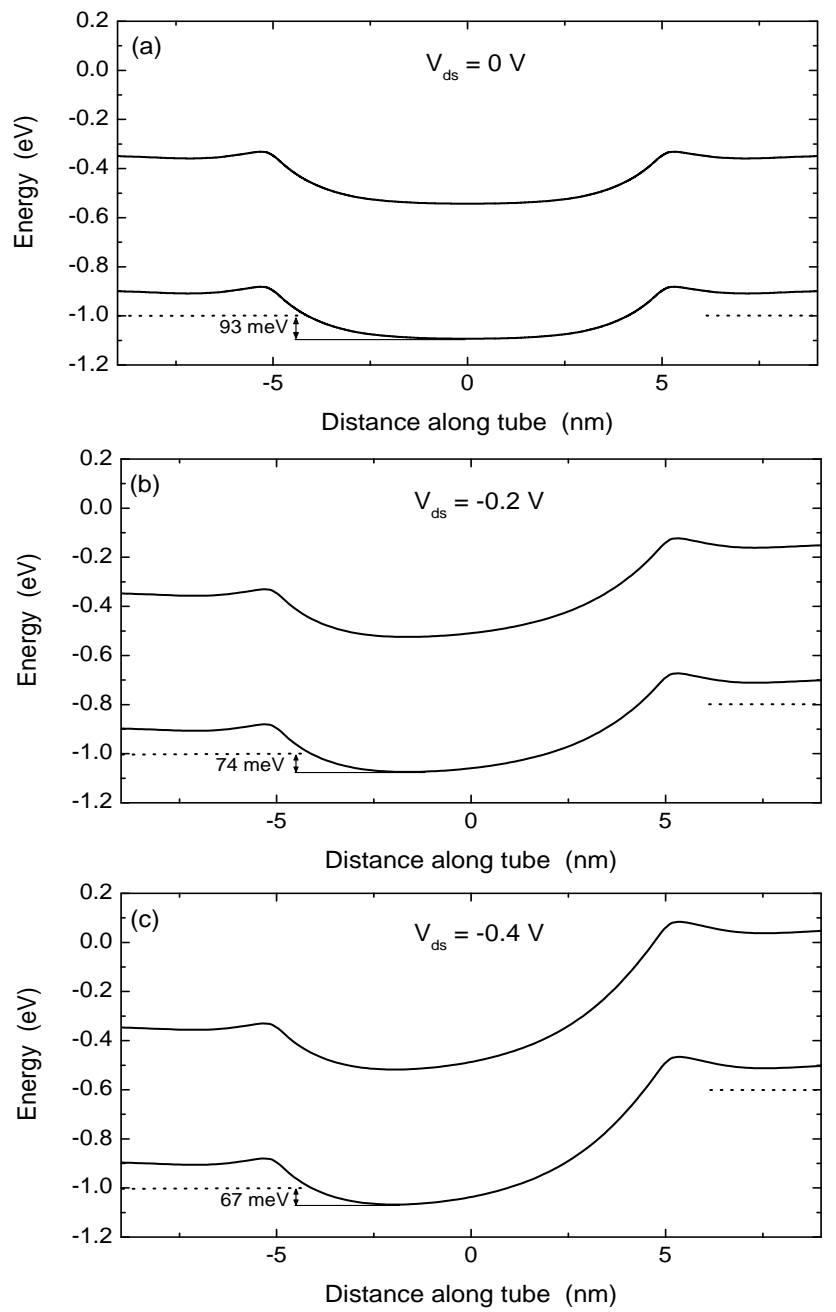

FIG. 7: Calculated self-consistent band bending for the $10 \mathrm{~nm}$ channel device for a gate-source voltage of $0.8 \mathrm{~V}$. The solid lines are the valence and conduction band edges; horizontal dotted lines are the metal Fermi levels. 

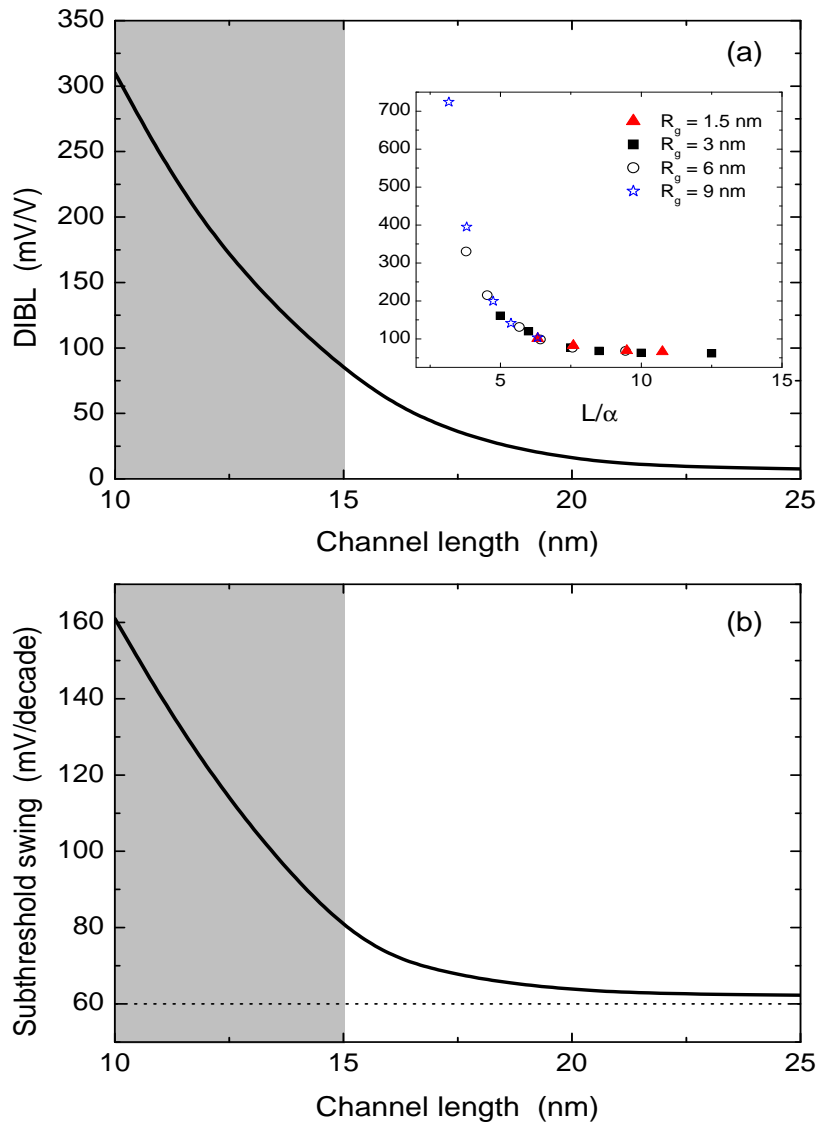

FIG. 8: Panels (a) and (b) show the variation of DIBL and subthreshold swing on channel length, respectively. Shaded areas are regions where the short channel effects are larger than typical device requirements. The inset in panel (a) shows collapse of the data for several devices of different channel lengths and gate radii upon scaling of the channel length. The horizontal dotted line in panel (b) is the theoretical limit for the subthreshold swing.

\section{TEMPERATURE DEPENDENCE OF ON STATE CONDUCTANCE}

As we discussed above, one of the important issues for carbon nanotubes is the nature of contacts between NTs and metals ${ }^{11,12,22}$. A useful way to elucidate the properties of contacts is to study the temperature dependence of the device characteristics ${ }^{4,23}$. In NT devices, temperature is useful to study the role of phonon scattering and Schottky barriers, as both of these lead to a temperature dependence of the conductance. We now show that even for the phonon-free, ballistic-ohmic NT device presented here, the conductance can have a strong temperature dependence. Figure 9 shows the calculated ON state conductance from our non-equilibrium Green's function approach as a function of temperature for $\Phi_{m}-\Phi_{N T}=$ $1 \mathrm{eV}$ and $\Phi_{m}-\Phi_{N T}=0.6 \mathrm{eV}$. Clearly, the conductance decreases with increasing temperature, with a stronger effect for the smaller metal workfunction. To understand the temperature dependence of the conductance $C$, we note that in the $\mathrm{ON}$ state the bands are flat with the Fermi level in the valence band, and we consider the expression $^{14}$

$$
C=\frac{4 e^{2}}{h} \int_{-\infty}^{\infty} P(E)\left(-\frac{\partial f}{\partial E}\right) d E
$$

where $P$ is the transmission probability and $f$ is the Fermi function. Assuming that $P(E)=1$ for $E<E_{v}=$ $E_{F}+\Delta$, we obtain

$$
C=\frac{4 e^{2}}{h} \frac{e^{\Delta / k T}}{1+e^{\Delta / k T}} .
$$

Thus, the conductance is sensitive to the self-consistent position of the metal Fermi level with respect to the valence band edge, $\Delta=E_{v}-E_{F}$. In principle, the value of $\Delta$ depends on the contact capacitance and temperature, and requires numerical evaluation at each temperature. But to illustrate the behavior, the dotted lines in Fig. 9 show the results of Eq. (18) for a constant $\Delta$ equal to its zero temperature value (we scaled the expression to fit the zero temperature conductance calculated numerically). Equation (18) indicates that the conductance can vary between the zero temperature limit of $4 e^{2} / h$ and the high temperature limit of $2 e^{2} / h$, with the variation occurring over a temperature range $k T \sim \Delta$. Higher workfunction metals should thus show a weaker dependence on temperature. Indeed, the metal with $\Phi_{m}-\Phi_{N T}=$ $1 \mathrm{eV}$ gives $\Delta \approx 0.1 \mathrm{eV}$ and its conductance varies more slowly than the metal with $\Phi_{m}-\Phi_{N T}=0.6 \mathrm{eV}$ that has $\Delta \approx 0.02 \mathrm{eV}$. The important point however is that the device conductance can vary with temperature by a factor of 2 even though there is no phonon scattering or Schottky barriers. Figure 9 also shows that recent experimental data ${ }^{4}$ for NT devices with Pd contacts agree well with the predicted behavior for $\Delta=0.02 \mathrm{eV}$.

\section{CONCLUSION}

There are several main conclusions from this work. First, for nanotubes embedded in metals, we have shown that charge transfer between the metal and the NT can renormalize the band lineup, and typically locates the Fermi level within a few tens of meV from the valence band edge. Second, our non-equilibrium quantum transport calculations indicate that nano-channel NT transistors can be dominated by short channel effects due to source-drain tunneling and drain-induced barrier lowering, even at channel lengths where Si transistors show good characteristics. Finally, for ohmic contacts, the ON state conductance decreases with increasing temperature, in contrast to Schottky barrier NT transistors.

Sandia is a multiprogram laboratory operated by Sandia Corporation, a Lockheed Martin Company, for the United States Department of Energy under contract DEAC01-94-AL85000. The Cornell Nanoscale Science and 


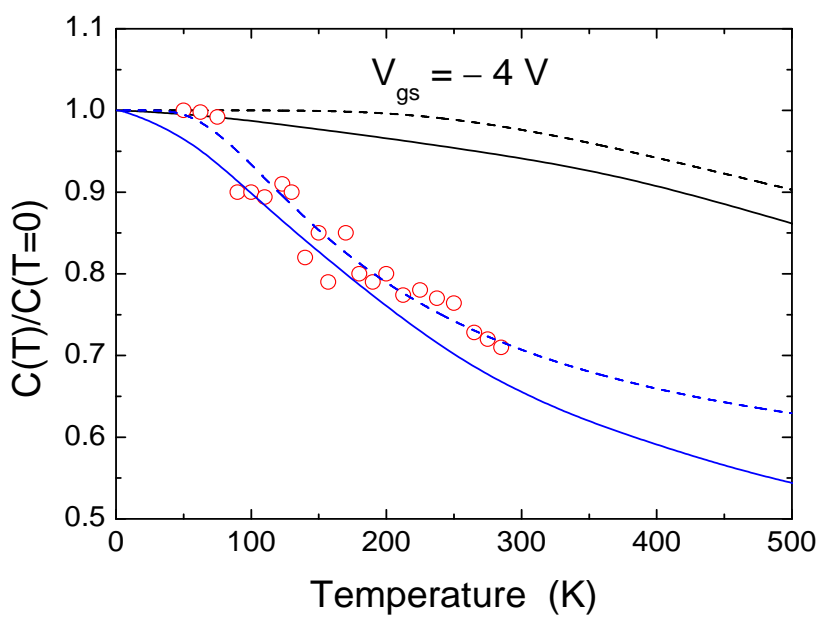

FIG. 9: Conductance of the nanotube transistor in the ON regime as a function of temperature. Black and blue solid lines are results of the atomistic calculations for $\Phi_{m}-\Phi_{N T}=1$ $\mathrm{eV}$ and $0.6 \mathrm{eV}$, respectively. Dotted lines are calculated from Eq. (18), see text for details. The circles are experimental data points extracted from Ref. ${ }^{4}$.
Technology Facility is part of the National Nanotechnology Infrastructure Network (NNIN) funded by the National Science Foundation.

*E-mail: fleonar@sandia.gov

${ }^{\dagger}$ E-mail: stewart@cnf.cornell.edu
${ }^{1}$ S. J. Tans, A. R. M. Verschueren, C. Dekker, Nature (London) 393, 49 (1998).

${ }^{2}$ R. Martel, T. Schmidt, H. R. Shea, T. Hertel, Ph. Avouris, Appl. Phys. Lett. 73, 2447 (1998).

3 B. M. Kim, T. Brintlinger, E. Cobas, M. S. Fuhrer, H. Zheng, Z. Yu, R. Droopad, J. Ramdani, K. Eisenberg, Appl. Phys. Lett. 84, 1946 (1998).

4 A. Javey, J. Guo, Q. Wang, M. Lundstrom, H. J. Dai, Nature (London) 424, 654 (2003).

${ }^{5}$ A. Javey, J. Guo, D. B. Former, Q. Wang, D. W. Wang, R. G. Gordon, M. Lundstrom, H. J. Dai, Nano Lett. 4, 447 (2004).

6 Y. Yaish, J.-Y. Park, S. Rosenblatt, V. Sazonova, M. Brink, P. L. McEuen, Phys. Rev. Lett. 92, 046401 (2004).

7 S. Heinze, J. Tersoff, R. Martel, V. Derycke, J. Appenzeller, Ph. Avouris, Phys. Rev. Lett. 89, 106801 (2002).

8 S. Heinze, M. Radosavljevic, J. Tersoff, Ph. Avouris, Phys. Rev. B 68, 235418 (2003).

9 F. Léonard and J. Tersoff, Phys. Rev. Lett. 88, 258302 (2002).

10 J. Guo, S. Datta, M. Lundstrom, IEEE Trans. Electron Devices 51, 172 (2004).

11 W. Kim, A. Javey, R. Tu, J. Cao, Q. Wang, H. Dai, Appl. Phys. Lett. 87, 173101 (2005).

12 Z. Chen, J. Appenzeller, J. Knoch, Y.-M. Lin, Ph. Avouris, Nano Lett. 5, 1497 (2005)
13 J. W. G. Wildöer, L. C. Venema, A. G. Rinzler, R. E. Smalley, C. Dekker, Nature (London) 391, 59 (1998); T.W. Odom, J. Huang, P. Kim, C. M. Lieber, Nature (London) 391, 62 (1998)

14 S. Datta, Electronic transport in mesoscopic systems (Cambridge University Press, Cambridge, England 1995).

15 M. P. Lopez-Sancho, J. M. Lopez-Sancho, J. Rubio, J. Phys. F. Met. Phys. 15, 851 (1985).

16 R. Lake, G. Klimeck, R. C. Bowen, D. Jovanovic, J. Appl. Phys. 81, 7845 (1997).

17 W. H. Press et al, Numerical recipes in fortran rry: The art of scientific computing, 2nd ed. (Cambridge University Press, Cambridge, England 1992).

18 F. Léonard and J. Tersoff, Phys. Rev. Lett. 83, 5174 (1999).

19 S.-H. Oh, D. Monroe, J. M. Hergenrother, IEEE Electron Device Lett. 21, 445 (2000).

20 D. L. John, C. Castro, D. L. Pulfrey, IEEE Trans. Nano. 2, 175 (2003).

${ }^{21}$ F. Léonard and J. Tersoff, Appl. Phys. Lett. 81, 4835 (2002).

22 F. Léonard and J. Tersoff, Phys. Rev. Lett. 84, 4693 (2000).

23 J. Appenzeller, M. Radosavljevic, J. Knoch, Ph. Avouris, Phys. Rev. Lett. 92, 048301 (2004). 